\title{
The use of a logical principle of uniform boundedness in analysis
}

\author{
Ulrich Kohlenbach \\ Department of Mathematics \\ University of Michigan \\ Ann Arbor \\ MI 48109, USA
}

December 1996

\section{Introduction}

This paper is part of a sequence of papers $([9],[10],[11],[12])$ resulting from our Habilitation thesis [8] addressing the following question:

What is the impact on the growth of extractable uniform bounds the use of various analytical principles $\Gamma$ in a given proof of an $\forall \exists$-sentence might have?

In particular we are interested in analyzing proofs of sentences having the form

$$
\text { (1) } \forall u^{1}, k^{0} \forall v \leq_{\rho} t u k \exists w^{0} A_{0}(u, k, v, w) \text {, }
$$

where $A_{0}$ is a quantifier-free formula ${ }^{1}$ (containing only $u, k, v, w$ as free variables) in the language of a suitable subsystem $\mathcal{T}^{\omega}$ of arithmetic in all finite types, $t$ is a closed term and $\leq_{\rho}$ is defined pointwise ( $\rho$ being an arbitrary finite type).

Lets suppose that (1) is provable in $\mathcal{T}^{\omega}$. Then from a given such proof one can extract an effective uniform bound $\Phi u k$ on $\exists w$, i.e.

$$
\text { (2) } \forall u^{1}, k^{0} \forall v \leq_{\rho} t u k \exists w \leq_{0} \Phi u k A_{0}(u, k, v, w),
$$

where the complexity (and in particular the growth) of $\Phi$ is limited by the complexity of the system $\mathcal{T}^{\omega}$ (see [7],[9]).

The predicate 'uniform' here means that the bound $\Phi$ does not depend on $v \leq_{\rho} t u k$.

In [7] we have shown, how sentences (1) naturally arise in analysis and why such uniform bounds are of numerical interest (see also [5],[6]).

Proofs in analysis can be formalized in a suitable base theory $\mathcal{T}^{\omega}$ plus certain (in general nonconstructive) analytical principles $\Gamma$ (typically not derivable in $\mathcal{T}^{\omega}$ ). In [9] a hierarchy of weak

\footnotetext{
${ }^{1}$ Throughout this paper $A_{0}, B_{0}, C_{0}, \ldots$ always denote quantifier-free formulas.
} 
subsystems $\mathrm{G}_{n} \mathrm{~A}^{\omega}$ of arithmetic in all finite types (whose definable type-1-objects correspond to the well-known Grzegorczyk hierarchy of functions) was introduced to calibrate faithfully the contribution of the use of $\Gamma$ to the growth of extractable bounds $\Phi$. The set of all closed terms of $G_{n} A^{\omega}$ is denoted by $\mathrm{G}_{n} \mathrm{R}^{\omega} . \mathrm{G}_{n} \mathrm{~A}_{i}^{\omega}$ is the intuitionistic variant of $\mathrm{G}_{n} \mathrm{~A}^{\omega}$ (for details see [9]).

We used monotone functional interpretation (introduced in [7]) as the basic proof-theoretic method to extract bounds $\Phi$ (given by closed term of $\mathrm{G}_{n} \mathrm{~A}^{\omega}$ ) from proofs

$$
\text { (3) } \mathrm{G}_{n} \mathrm{~A}^{\omega}+\Delta+\mathrm{AC}-\mathrm{qf} \vdash(1)
$$

where

$$
\mathrm{AC}^{\rho, \tau}-\mathrm{qf}: \forall x^{\rho} \exists y^{\tau} A_{0}(x, y) \rightarrow \exists Y^{\tau \rho} \forall x^{\rho} A_{0}(x, Y x) \text { and } \mathrm{AC}-\mathrm{qf}:=\bigcup_{\rho, \tau \in \mathbf{T}}\left\{\left(\mathrm{AC}^{\rho, \tau}-\mathrm{qf}\right)\right\}
$$

is the schema of choice for quantifier-free formulas and $\Delta$ is a set of 'axioms' having the form

$$
\text { (4) } \forall x^{\delta} \exists y \leq_{\rho} s x \forall z^{\tau} G_{0}(x, y, z) \text {, }
$$

where $G_{0}$ is a quantifier-free formula containing only $x, y, z$ free and $s$ is a closed term.

In particular for $n=2$ (resp. $n=3$ ) one can extract a bound $\Phi u k$ which is a polynomial (resp. a finitely iterated exponential function) in $u^{M} x:=\max _{i \leq x} u(i)$ and $k$ (see [9] for details).

For suitable $\Delta$ already $\mathrm{G}_{2} \mathrm{~A}^{\omega}+\Delta+\mathrm{AC}-\mathrm{qf}$ covers a substantial part of standard analysis (see [8], [9]).

In [9] also we introduced new axioms $F$ and $F^{-}$which both (essentially) have the form (4) and are true in the type structure of all strongly majorizable functionals (see [1]) but are false in the full set-theoretic model (weaker versions of these axioms were studied already in [7]). Thus, whereas $F, F^{-}$do not contribute to the construction of bounds extracted from a proof, the verification of these bounds so long uses these axioms. However $F^{-}$can be eliminated from the verification proof by further proof-theoretic transformations (which do not effect the bounds themselves) so that the bounds extracted can also be verified without $F^{-}$. For bounds of type $\leq 1$ this is also possible for proofs using $F$. The importance of the $F, F^{-}$rests on the fact that they imply combined with $\mathrm{AC}^{1,0_{-}}$ qf (which also has a trivial monotone functional interpretation) relative to $\mathrm{G}_{2} \mathrm{~A}^{\omega}$ strong principles of uniform boundedness $\Sigma_{1}^{0}-\mathrm{UB}, \Sigma_{1}^{0}-\mathrm{UB}^{-}$(see definitions $2.5,2.11$ below) which prove many important analytical theorems in their direct formulation (i.e. without any special representation) which do not have the form (4) by themselves: In this paper we show that $\Sigma_{1}^{0}-\mathrm{UB}^{-}$implies

1) Every pointwise continuous function $G:[0,1]^{d} \rightarrow \mathbb{R}$ is uniformly continuous on $[0,1]^{d}$ and possesses a modulus of uniform continuity.

2) $[0,1]^{d} \subset \mathbb{R}^{d}$ has the (sequential form of the) Heine-Borel covering property.

3) Dini's theorem: Every sequence $G_{n}$ of pointwise continuous functions : $[0,1]^{d} \rightarrow \mathbb{R}$ which increases pointwise to a pointwise continuous function $G:[0,1]^{d} \rightarrow \mathbb{R}$ converges uniformly on $[0,1]^{d}$ to $G$ and there exists a modulus of uniform convergence.

4) Every strictly increasing pointwise continuous function $G:[0,1] \rightarrow \mathbb{R}$ possesses a uniformly continuous inverse function $G^{-1}:[G 0, G 1] \rightarrow[0,1]$ together with a modulus of uniform continuity. 
As a consequence of this we obtain the following result: If $\forall x^{0} \forall y \leq_{\rho} s x \exists z^{0} A_{0}(x, y, z)$ is proved in $\mathrm{G}_{2} \mathrm{~A}^{\omega}+\mathrm{AC}-\mathrm{qf}$ plus axioms having the form (4) plus the theorems 1)-4), then one can extract from the proof a polynomial $p$ which provides a uniform bound on ' $\exists z$ ' (which does not depend on $y$ ), i.e. $\forall x^{0} \forall y \leq_{\rho} s x \exists z \leq_{0} p(x) A_{0}(x, y, z)$. If instead of $x^{0}$ we have $x^{0}, \tilde{x}^{1}$, one obtains a polynomial relatively to $x$ and $\tilde{x}^{M}$, where $\tilde{x}^{M} k:=\max (\tilde{x} 0, \ldots, \tilde{x} k)$ (in the sense of [9]).

It is well-known that the existence of a function $G:[0,1]^{d} \rightarrow \mathbb{R}$, represented by a functional $\Phi^{1(1)}$, which is not continuous can be proved only by an instance of arithmetical comprehension over functions

$$
C A_{a r}^{1}: \equiv \exists \Phi^{0(1)} \forall f^{1}\left(\Phi f={ }_{0} 0 \leftrightarrow A(f)\right) \text {, where } A \text { is an arithmetical formula. }
$$

Since we deal with theories which do not contain $C A_{a r}^{1}$ it is consistent to assume as an axiom that all functions $G:[0,1]^{d} \rightarrow \mathbb{R}$, which are given explicitly by a functional $\Phi^{1(1)}$, are uniformly continuous. ${ }^{2}$ This is achieved by the axiom $F$ :

$\mathrm{G}_{2} \mathrm{~A}^{\omega}+F+\mathrm{AC}^{1,0}{ }_{\text {qf }}$ proves: Every function $G:[0,1]^{d} \rightarrow \mathbb{R}$ is uniformly continuous and possesses a modulus of uniform continuity.

The use of $F$ (which does not contribute to the bounds extracted) has the nice property that continuous functions $G:[0,1]^{d} \rightarrow \mathbb{R}$ are nothing else than functionals $\Phi^{1(1)}$ which are extensional w.r.t. $=_{[0,1]^{d}}$ and $=_{\mathbb{R}}$ (and thus represent a function : $[0,1]^{d} \rightarrow \mathbb{R}$ ). This simplifies the formalization of given proofs and thereby the extraction of bounds from these proofs. Moreover the proofs of 1)-4) (which now hold for arbitrary functions $G, G_{n}$ ) become more simple.

From the work on the program of so-called 'reverse mathematics' (see [3],[16]) it is known that e.g. 1) and 2) when suitably encoded are provable in a subsystem $\mathrm{RCA}_{0}+\mathrm{WKL}$ of second-order arithmetic which is based on the binary König's lemma and $\Sigma_{1}^{0}$-induction ${ }^{3}$. The provably recursive functions of $\mathrm{RCA}_{0}+\mathrm{WKL}$ are just the primitive recursive ones. This was firstly proved by H. Friedman in 1979 (in an unpublished paper) using model-theoretic methods. Later on W.Sieg gave a prooftheoretic treatment of this result using cut-elimination (see [14]). In [4] we proved the conservativity of WKL over the finite type theories $\mathrm{PRA}^{\omega}$ and $\mathrm{PA}^{\omega}$ even for higher type sentences $\forall x^{1} \forall y \leq \rho$ $s x \exists z^{\tau} A_{0}(x, y, z)$, where $\rho, \tau$ are arbitrary types. Moreover we gave a perspicuous method for the the extraction of bounds from proofs using WKL and arbitrary axioms (4) by a new combination of functional interpretation with majorization which, in [7], was simplified even further to the monotone functional interpretation. In [5], [6] this was applied to concrete proofs in best approximation theory yielding new numerical estimates which improved known estimates significantly (see [7] for a discussion of these results). In [5] we also gave a detailed representation of $\mathbb{R}, C[0,1]$ and more general complete separable metric spaces and showed that e.g. the theorem stating the attainment of the maximum value for $f \in C\left([0,1]^{d}, \mathbb{R}\right.$ ) (for $d=1$ ) as well as some more specific theorems from approximation theory have the logical form (4). However we did not determine the growth of the terms needed in the formalization of these theorems as axioms (4). Only by our much more involved

\footnotetext{
${ }^{2}$ The restriction 'given explicitly by a functional' is essential. Of course we can formulate functional dependencies in e.g. $\mathrm{G}_{2} \mathrm{~A}^{\omega}$ which describe a discontinuous functional: E.g. we can prove

$\forall f^{1} \exists ! x^{0}([\exists y(f y=0) \rightarrow f x=0 \wedge \forall \tilde{x}<x(f \tilde{x} \neq 0)] \wedge[\forall y(f y \neq 0) \rightarrow x=0])$ and $x$ does not depend continuously on $f$ but we cannot show the existence of a functional $\Phi^{0(1)}$ which maps $f$ to $x$.

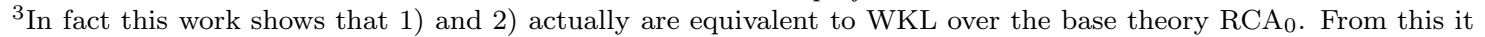
follows that these theorems have no functional interpretation in Gödel's T.
} 
representation of $C[0,1]$ and its generalization to $C\left([0,1]^{d}, \mathbb{R}\right)$ and the explicit definition of the basic function(al)s of analysis in the chapters 3 and 5 of [8] we were able to show that these principles can be expressed as axioms (4) in $\mathbf{G}_{2} \mathbf{A}^{\omega}$.

Since 1)-4) do not have the logical form (4) one has to consider their proofs. The proofs of 1) and 2) using WKL (relative to $\mathrm{RCA}_{0}$ ) require a tedious coding technique. In particular pointwise continuous functions have to be coded as a complicated set of quadruples of rational numbers (see [15]). Although working in the more flexible language of finite types makes it much easier to speak about such functions (namely as certain functionals of type 1(1)) this does not help as long as one has to use WKL as the basic principle of proof. In fact even the formulation of WKL itself uses the coding of sequences of variable length and therefore cannot be carried out in $G_{2} A^{\omega}$. The motivation for our axioms $F, F^{-}$was to formulate a more general higher type version of WKL which can be formulated and applied without the need of coding up objects like functions $[0,1] \rightarrow \mathbb{R}$. This allows very short proofs for 1)-4) in $\mathrm{G}_{2} \mathrm{~A}^{\omega}+F^{-}+\mathrm{AC}^{1,0}-\mathrm{qf}$. The relationship between $F^{-}$and WKL is studied in detail in [9].

All undefined notions are used in the sense of [9] on which this paper relies. Furthermore, in section 3 we refer to the representation of $\mathbb{R}^{d}$ and $[0,1]^{d}$ developed in [12].

\section{The axiom $F$ and the principle of uniform boundedness}

In [9] we introduced the following 'non-standard' axiom $F$ which is not valid in the full set-theoretic type structure $\mathcal{S}^{\omega}$ of all set-theoretic functionals but is true in the type structure of all strongly majorizable functionals $\mathcal{M}^{\omega}$ which was introduced in [1] for different purposes (see [9] for details; a special case of $F$-called $F_{0}$ in [9]- was already studied in [7]). In this section we review some of the results on $F$ from [9].

Definition 2.1 $\boldsymbol{F}: \equiv \forall \Phi^{2(0)}, y^{1(0)} \exists y_{0} \leq_{1(0)} y \forall k^{0} \forall z \leq_{1} y k\left(\Phi k z \leq_{0} \Phi k\left(y_{0} k\right)\right)$.

$F$ implies the existence of a sequence of bounds for a sequence $\Phi^{2(0)}$ of type-2-functionals on a sequence of fan's:

Proposition $2.2 G_{1} A_{i}^{\omega} \vdash F \rightarrow \forall \Phi^{2(0)}, y^{1(0)} \exists \chi^{1} \forall k^{0} \forall z \leq_{1} y k\left(\Phi k z \leq_{0} \chi k\right)$.

Proof: Put $\chi k:=\Phi\left(y_{0} k\right) k$ for $y_{0}$ from $F$.

Using the extensionality axiom $(E)$ (rather than the quantifier-free rule of extensionality from $\mathrm{G}_{n} \mathrm{~A}^{\omega}$ ) $F$ can be transformed into a sentence $\tilde{F}$ having the logical form $\forall x \exists y \leq s x \forall z A_{0}$ of an axiom $\Delta$ (because of the universal quantifier hidden in $z \leq_{1} y k, F$ itself does not have this form):

Lemma $2.3 E-G_{1} A_{i}^{\omega} \vdash F \leftrightarrow \widetilde{F}: \equiv \forall \Phi^{2(0)}, y^{1(0)} \exists y_{0} \leq_{1(0)} y \forall k^{0}, z^{1}\left(\Phi k\left(\min _{1}(z, y k)\right) \leq_{0} \Phi k\left(y_{0} k\right)\right)$.

Proof: ' $\rightarrow$ ' is trivial. ' $\leftarrow$ ' follows from $z \leq_{1} y k \rightarrow \min _{1}(z, y k)=_{1} z$ by the use of the extensionality axiom (E).

In the following $\mathrm{b}-\mathrm{AC}:=\bigcup_{\delta, \rho \in \mathbf{T}}\left\{\left(\mathrm{b}-\mathrm{AC}^{\delta, \rho}\right)\right\}$ denotes the schema

$$
\left(\mathrm{b}-\mathrm{AC}^{\delta, \rho}\right): \forall Z^{\rho \delta}\left(\forall x^{\delta} \exists y \leq_{\rho} Z x A(x, y, Z) \rightarrow \exists Y \leq_{\rho \delta} Z \forall x A(x, Y x, Z)\right) .
$$


Because of the lemma above we can treat $F$ as an axiom $\in \Delta$ in the presence of (E). In order to apply our monotone functional interpretation one firstly has to eliminate (E) from the proof. In [9] the following result on $F$ is established:

Theorem 2.4 ([9]) Assume that $n \geq 1$. Let $\Delta$ be a set of sentences having the form $\forall u^{\gamma} \exists v \leq{ }_{\delta} t u \forall w^{\eta} B_{0}$, where $t \in G_{n} R^{\omega}$ and $\gamma, \eta \leq 2, \delta \leq 1$ such that $\mathcal{S}^{\omega} \models \Delta$. Furthermore let $s \in$ $G_{n} R^{\omega}$ and $A_{0} \in \mathcal{L}\left(G_{n} A^{\omega}\right)$ be a quantifier-free formula containing only $x, y, z$ free and let $\alpha, \beta \in \mathbf{T}$ such that $(\alpha=0 \wedge \beta \leq 1)$ or $(\alpha=1 \wedge \beta=0)$, and $\tau \leq 2$. Then the following rule holds:

$$
\left\{\begin{array}{l}
E-G_{n} A^{\omega}+F+\Delta+A C^{\alpha, \beta}-q f \vdash \forall x^{1} \forall y \leq_{1} s x \exists z^{\tau} A_{0}(x, y, z) \\
\Rightarrow \text { by elimination of (E). neg. transl. and monotone functional interpretation } \exists \Psi \in G_{n} R^{\omega}: \\
G_{n} A_{i}^{\omega}+\tilde{F}+\Delta+b-A C \vdash \forall x^{1} \forall y \leq_{1} s x \exists z \leq_{\tau} \Psi x A_{0}(x, y, z) \\
\text { and } \mathcal{M}^{\omega}, \mathcal{S}^{\omega} \models \forall x^{1} \forall y \leq_{1} s x \exists z \leq_{\tau} \Psi x A_{0}(x, y, z) .
\end{array}\right.
$$

$\Psi$ is built up from $0^{0}, 1^{0}, \max _{\rho}, \Phi_{1} f x:=\max (f 0, \ldots, f x)$ and majorizing terms $s^{4}$ for the terms $t$ occurring in the quantifier axioms $\forall x G x \rightarrow G t$ and $G t \rightarrow \exists x G x$ which are used in the given proof by use of $\lambda$-abstraction and substitution.

(An analogous result holds for $E-P R A^{\omega}, E-P A^{\omega}$ with $\Psi \in \widehat{\mathrm{PR}}^{\omega}$ resp. $\Psi \in T$ ).

Note that the conclusion of theorem 2.4 is valid in $\mathcal{S}^{\omega}$ although $F$ is not.

Our applications of $F$ actually are based on the following consequence of $F+\mathrm{AC}^{1,0}-\mathrm{qf}$ :

Definition 2.5 The schema of uniform $\Sigma_{1}^{0}$-boundednes is defined as

$$
\Sigma_{1}^{0}-\mathbf{U B}: \equiv\left\{\begin{aligned}
\forall y^{1(0)}\left(\forall k^{0} \forall x \leq_{1} y k \exists z^{0} A(x, y, k, z)\right. \\
\left.\rightarrow \exists \chi^{1} \forall k^{0} \forall x \leq_{1} y k \exists z \leq_{0} \chi k A(x, y, k, z)\right),
\end{aligned}\right.
$$

where $A \equiv \exists \underline{l} A_{0}(\underline{l})$ and $\underline{l}$ is a tuple of variables of type 0 and $A_{0}$ is a quantifier-free formula (which may contain parameters of arbitrary types).

Proposition 2.6 ([9]) Assume that $n \geq 2$. Then $G_{n} A^{\omega}+A C^{1,0}-q f \vdash F \rightarrow \Sigma_{1}^{0}-U B$.

Proof: $\forall k^{0} \forall x^{1} \leq_{1} y k \exists z^{0} A(x, y, k, z)$ implies $\forall k^{0} \forall x^{1} \exists z^{0}, v^{0}\left(x v \leq_{0} y k v \rightarrow A(x, y, k, z)\right)$. Thus using the fact that $k, x$ as well as $z, v, \underline{l}$ can be coded together in $\mathrm{G}_{2} \mathrm{~A}^{\omega}$, one obtains by $\mathrm{AC}^{1,0}{ }^{\mathrm{q}}$ - $\mathrm{f}$ the existence of a functional $\Phi^{2(0)}$ such that $\forall k^{0} \forall x \leq_{1} y k A(x, y, k, \Phi k x)$. By proposition 2.2 we finally obtain $\exists \chi^{1} \forall k^{0} \forall x \leq_{1} y k\left(\chi k \geq_{0} \Phi k x\right)$.

In [9] we have shown that $\Sigma_{1}^{0}$-UB together with classical logic implies the existence of a modulus of uniform continuity for each extensional $\Phi^{1(1)}$ on $\left\{z^{1}: z \leq_{1} y\right\}$ (where 'continuity' refers to the usual metric on the Baire space $\mathbb{N}^{\mathbb{N}}$ ):

Proposition 2.7 For $n \geq 2$ the following holds

$$
\begin{aligned}
& G_{n} A^{\omega}+\Sigma_{1}^{0}-U B \vdash \\
& \forall \Phi^{1(1)}\left(\operatorname{ext}(\Phi) \rightarrow \forall y^{1} \exists \chi^{1} \forall k^{0} \forall z_{1}, z_{2} \leq_{1} y\left(\bigwedge_{i \leq_{0} \chi^{k}}\left(z_{1} i={ }_{0} z_{2} i\right) \rightarrow \bigwedge_{j \leq 0}\left(\Phi z_{1} j={ }_{0} \Phi z_{2} j\right)\right)\right),
\end{aligned}
$$

\footnotetext{
${ }^{4}$ Here $t^{*}[\underline{a}]$ is called a majorizing term if $\lambda \underline{a} . t^{*} \mathrm{~s}-\mathrm{maj} \lambda \underline{a} . t$, where $\underline{a}$ are all free variables of $t$.
} 
where $\operatorname{ext}(\Phi): \equiv \forall z_{1}^{1}, z_{2}^{1}\left(z_{1}={ }_{1} z_{2} \rightarrow \Phi z_{1}={ }_{1} \Phi z_{2}\right)$

For many applications (in particular for most of the ones given in the present paper) a weaker version $F^{-}$of $F$ is sufficient which we also have studied in [9] for the following reasons:

1) $F^{-}$has directly the logical form $\forall x \exists y \leq s x \forall z A_{0}$ of an axiom $\in \Delta$. Thus a transformation like $F \mapsto \tilde{F}$ is not needed. This simplifies the extraction of bounds and allows the generalization to higher types (see thm.2.13 below).

2) There is a simple purely syntactical way to eliminate $F^{-}$from the proof for the verification of the bound extracted (see thm.2.13) thereby yielding a verification in $\mathrm{G}_{\max (3, n)} \mathrm{A}_{i}^{\omega}$. In particular no relativization to $\mathcal{M}^{\omega}$ is needed. For $F$ a much more complicated elimination yielding a verification only in $\mathrm{HA}^{\omega}$ and only for $\tau \leq 1$ and without $\Delta$ in thm.2.4 is given in [7]. Recently we have succeeded to extend the elimination procedure for $F^{-}$to $F$ however the verification then needs $\mathrm{PA}^{\omega}$ plus a restricted form of b-AC.

\section{Definition 2.8}

$F^{-}: \equiv \forall \Phi^{2(0)}, y^{1(0)} \exists y_{0} \leq_{1(0)} y \forall k^{0}, z^{1}, n^{0}\left(\bigwedge_{i<0 n}\left(z i \leq_{0} y k i\right) \rightarrow \Phi k(\overline{z, n}) \leq_{0} \Phi k\left(y_{0} k\right)\right)$, where, for $z^{\rho 0}$, $(\overline{z, n})\left(k^{0}\right):={ }_{\rho} z k$, if $k<_{0} n$ and $:=0^{\rho}$, otherwise (It is clear that $\lambda z, n \cdot(\overline{z, n}) \in G_{2} R^{\omega}$ ).

Remark 2.9 $\mathrm{F}^{-}$is a weakening of $F$ (to 'finite' sequences) and therefore is also true in $\mathcal{M}^{\omega}$. However $F^{-}$still does not hold in $\mathcal{S}^{\omega}$ (see [9]).

Lemma $2.10 G_{1} A_{i}^{\omega} \vdash F^{-} \rightarrow \forall \Phi^{2(0)}, y^{1(0)} \exists \chi^{1(0)} \forall k^{0}, z^{1}, n^{0}\left(\bigwedge_{i<0} n\left(z i \leq_{0} y k i\right) \rightarrow \Phi k(\overline{z, n}) \leq_{0} \chi k\right)$.

Definition 2.11 The schema $\Sigma_{1}^{0}-U B^{-}$is the following weakening of $\Sigma_{1}^{0}-U B$ :

$$
\boldsymbol{\Sigma}_{\mathbf{1}}^{\mathbf{0}-\mathbf{U B}^{-}}: \equiv\left\{\begin{array}{r}
\forall y^{1(0)}\left(\forall k^{0} \forall x \leq_{1} y k \exists z^{0} A(x, y, k, z) \rightarrow \exists \chi^{1} \forall k^{0}, x^{1}, n^{0}\right. \\
\left.\left(\bigwedge_{i<_{0} n}\left(x i \leq_{0} y k i\right) \rightarrow \exists z \leq_{0} \chi k A((\overline{x, n}), y, k, z)\right)\right)
\end{array}\right.
$$

where $A \in \Sigma_{1}^{0}$ as in $\Sigma_{1}^{0}-U B$.

Proposition 2.12 ([9]) For each $n \geq 2$ we have $G_{n} A^{\omega}+A C^{1,0}-q f \vdash F^{-} \rightarrow \Sigma_{1}^{0}-U B^{-}$.

Theorem 2.13 ([9]) Assume $n \geq 1, \tau \leq 2, s \in G_{n} R^{\omega}$. Let $A_{0}(x, y, z) \in \mathcal{L}\left(G_{n} A^{\omega}\right)$ be a quantifierfree formula containing only $x, y, z$ as free variables. Then the following rule holds:

$$
\left\{\begin{array}{l}
G_{n} A^{\omega} \oplus A C-q f \oplus F^{-} \vdash \forall x^{1} \forall y \leq_{\rho} s x \exists z^{\tau} A_{0}(x, y, z) \\
\Rightarrow \text { by neg. transl. and monotone functional interpretation } \exists \Psi \in G_{n} R^{\omega} \text { such that } \\
G_{\max (3, n)} A_{i}^{\omega} \vdash \forall x^{1} \forall y \leq_{\rho} s x \exists z \leq_{\tau} \Psi x A_{0}(x, y, z) .
\end{array}\right.
$$

$\Psi$ is built up from $0^{0}, 1^{0}, \max _{\rho}, \Phi_{1} f x:=\max (f 0, \ldots, f x)$ and majorizing terms for the terms $t$ occurring in the quantifier axioms $\forall x G x \rightarrow G t$ and $G t \rightarrow \exists x G x$ which are used in the given proof 
by use of $\lambda$-abstraction and substitution..$^{5}$

For $\rho \leq 1, G_{n} A^{\omega} \oplus A C-q f \oplus F^{-}$can be replaced by $E-G_{n} A^{\omega}+A C^{\alpha, \beta}-q f+F^{-}$, where $\alpha, \beta$ are as in thm.2.4. Furthermore on may add axioms $\Delta$ (having the form as in theorem2.4) to

$G_{n} A^{\omega} \oplus A C-q f \oplus F^{-}$. Then the conclusion holds in $G_{\max (3, n)} A_{i}^{\omega}+\Delta+b-A C$. For $n=2$ and $\tau=0$, $\Psi x$ is a polynomial in $x^{M}(i):=\max _{j \leq i} x(j)$.

An analogous result holds for $P R A^{\omega}$ and $P A^{\omega}$ with $\Psi \in \widehat{P R}^{\omega}$ resp. $\in T$ and verification in $P R A_{i}^{\omega}$ resp. $P A_{i}^{\omega}$.

In [9] we showed that $F^{-}$implies (relatively to $\mathrm{G}_{1} \mathrm{~A}^{\omega}+\mathrm{AC}^{1,0}-\mathrm{qf}$ ) a generalization of the binary ('weak') König's lemma WKL:

Definition 2.14 (Troelstra(74))

$W K L: \equiv \forall f^{1}\left(T(f) \wedge \forall x^{0} \exists n^{0}\left(\right.\right.$ lth $\left.\left.n={ }_{0} x \wedge f n={ }_{0} 0\right) \rightarrow \exists b \leq_{1} \lambda k .1 \forall x^{0}\left(f(\bar{b} x)=_{0} 0\right)\right)$, where $T f: \equiv \forall n^{0}, m^{0}\left(f(n * m)=_{0} 0 \rightarrow f n=_{0} 0\right) \wedge \forall n^{0}, x^{0}\left(f(n *\langle x\rangle)=_{0} 0 \rightarrow x \leq_{0} 1\right)$

(i.e. $T(f)$ asserts that $f$ represents a 0,1 -tree).

Let $\mathrm{WKL}_{\text {seq }}^{2}$ denote the following sequential version of $\mathrm{WKL}$, which states that for every sequence of infinite 0,1 -trees there exists a sequence of infinite branches and represents binary trees by functionals of finite types thereby avoiding the coding functional $\bar{f} x=\langle f 0, \ldots, f(x-1)\rangle$ which is available in $\mathrm{G}_{n} \mathrm{~A}^{\omega}$ only for $n \geq 3$ (whereas the case $n=2$ where polynomial bounds are extractable is of particular mathematical relevance).

Definition 2.15

$$
W K L_{\text {seq }}^{2}: \equiv\left\{\begin{aligned}
\forall \Phi^{0010}\left(\forall k^{0}, x^{0} \exists b \leq_{1} \lambda n^{0} \cdot 1^{0} \bigwedge_{i=0}^{x}\left(\Phi k(\overline{b, i}) i==_{0} 0\right)\right. \\
\left.\rightarrow \exists b \leq_{1(0)} \lambda k^{0}, n^{0} .1 \forall k^{0}, x^{0}\left(\Phi k(\overline{b k, x}) x={ }_{0} 0\right)\right) .
\end{aligned}\right.
$$

Proposition 2.16 ([9]) $G_{3} A_{i}^{\omega} \vdash W K L_{\text {seq }}^{2} \rightarrow W K L$.

Theorem 2.17 ([9]) $G_{2} A^{\omega}+A C^{0,1}-q f \vdash \Sigma_{1}^{0}-U B^{-} \rightarrow W K L_{\text {seq }}^{2}$.

Together with (the proof of) prop.2.12 this theorem implies the following

Corollary $2.18([9])$ Let $n \geq 2$. Then $\left(G_{n} A^{\omega}+A C^{1,0}-q f+A C^{0,1}-q f\right) \oplus F^{-} \vdash W K L_{\text {seq }}^{2}$. Hence theorem 2.4 and theorem 2.13 capture proofs using $W K L_{\text {seq }}^{2}$. Moreover we have the following rule

$$
\left\{\begin{array}{l}
E-G_{2} A^{\omega}+A C^{\alpha, \beta}-q f+W K L_{s e q}^{2} \vdash \forall x^{0} \forall y \leq_{1} s x \exists z^{0} A_{0}(x, y, z) \\
\Rightarrow \exists(e f f .) k, c_{1}, c_{2} \in \mathbb{N} \text { such that } \\
G_{3} A_{i}^{\omega} \vdash \forall x^{0} \forall y \leq_{1} s x \exists z \leq_{0} c_{1} x^{k}+c_{2} A_{0}(x, y, z),
\end{array}\right.
$$

where $s \in G_{2} R^{\omega}$ and $A_{0}$ is a quantifier-free formula of $G_{2} A^{\omega}$ which contains only $x, y, z$ as free variables and $(\alpha=0 \wedge \beta \leq 1)$ or $(\alpha=1 \wedge \beta=0)$.

See [9] for a discussion of the relationship between $F^{-}$and $\mathrm{WKL}_{\text {seq }}^{2}$.

\footnotetext{
${ }^{5}$ Here $\oplus$ means that $F^{-}$and $\mathrm{AC}-$ qf must not be used in the proof of the premise of an application of the quantifierfree rule of extensionality $\mathrm{QF}-\mathrm{ER} . \mathrm{G}_{n} \mathrm{~A}^{\omega}$ satisfies the deduction theorem w.r.t $\oplus$ but not w.r.t + . In fact the theorem also holds for $\left(\mathrm{G}_{n} \mathrm{~A}^{\omega}+\mathrm{AC}-\mathrm{qf}\right) \oplus F^{-}$since the deduction property is used in its proof only for $F^{-}$.
} 


\section{Applications of $\Sigma_{1}^{0}-\mathrm{UB}$ (resp. $\Sigma_{1}^{0}-\mathrm{UB}^{-}$) relatively to $\mathrm{G}_{2} \mathbf{A}^{\omega}$}

In this section we show how $\Sigma_{1}^{0}-\mathrm{UB}$ and $\Sigma_{1}^{0}-\mathrm{UB}^{-}$can be used in analysis:

\section{Application 1:}

Proposition 3.1 For every fixed number $d$ the following holds:

1) $G_{2} A^{\omega} \oplus \Sigma_{1}^{0}-U B$ (and therefore $G_{2} A^{\omega} \oplus A C^{1,0}-q f \oplus F$ ) proves:

Every function $F:[0,1]^{d} \rightarrow \mathbb{R}$ is uniformly continuous and possesses a modulus of uniform continuity.

2) $G_{2} A^{\omega} \oplus \Sigma_{1}^{0}-U B^{-}$(and therefore $G_{2} A^{\omega} \oplus A C^{1,0}-q f \oplus F^{-}$) proves:

Every pointwise continuous function $F:[0,1]^{d} \rightarrow \mathbb{R}$ is uniformly continuous and possesses a modulus of uniform continuity.

Proof: 1) Formulated in $\mathrm{G}_{2} \mathrm{~A}^{\omega}$ the assertion above reads as follows:

If $\Phi^{1(1)}$ (note that we do not need the complicated representation of $C\left([0,1]^{d}, \mathbb{R}\right.$ ) from chapter 3 ) of $[8]$ represents a function $[0,1]^{d} \rightarrow \mathbb{R}$, i.e.

$\forall x_{1}^{1}, x_{2}^{1}\left(\bigwedge_{i=1}^{d}\left(0 \leq_{\mathbb{R}} \nu_{i}^{d}\left(x_{1}\right), \nu_{i}^{d}\left(x_{2}\right) \leq_{\mathbb{R}} 1 \wedge \nu_{i}^{d}\left(x_{1}\right)==_{\mathbb{R}} \nu_{i}^{d}\left(x_{2}\right)\right) \rightarrow \Phi x_{1}={ }_{\mathbb{R}} \Phi_{2}\right)$, then $\Phi$ is uniformly continuous on $[0,1]^{d}$ and possesses a modulus of uniform continuity (Here $\nu^{d}, \nu_{i}^{d}$ are the coding functions for $d$-tuples of number-theoretic functions from [9]). By the representation of $[0,1]$ from [12] we can restrict ourselves to representatives $x^{1}$ of elements of $[0,1]^{d}$ which satisfy $\nu_{i}^{d}(x) \leq_{1} M$ for $i=1, \ldots, d$ (where $M:=\lambda n \cdot j(6(n+1), 3(n+1)-1))$.

$$
\forall x_{1}, x_{2} \leq_{1} \nu^{d}(M, \ldots, M)\left(\bigwedge_{i=1}^{d}\left(\widetilde{\nu_{i}^{d}\left(x_{1}\right)}={ }_{\mathbb{R}} \widetilde{\nu_{i}^{d}\left(x_{2}\right)}\right) \rightarrow \Phi \tilde{x}_{1}=\mathbb{R} \Phi \tilde{x}_{2}\right)
$$

is equivalent to ${ }^{6}$

$$
\forall x_{1}, x_{2} \leq_{1} \nu^{d}(M, \ldots, M) \forall k^{0} \exists n^{0}(\underbrace{\left\|\tilde{x}_{1}-\mathbb{R}_{\mathbb{R}^{d}} \tilde{x}_{2}\right\|_{\max } \leq_{\mathbb{R}} \frac{1}{n+1} \rightarrow\left|\Phi \tilde{x}_{1}-\mathbb{R} \Phi \tilde{x}_{2}\right|_{\mathbb{R}}<_{\mathbb{R}} \frac{1}{k+1}}_{\equiv: A \in \Sigma_{1}^{0}}),
$$

where $\|\cdot\|_{\max }$ denotes the maximum metric $^{7}$ on $\mathbb{R}^{d}$.

Since $x_{1}, x_{2}$ can be coded together, $\Sigma_{1}^{0}$-UB yields (using the monotonicity of $A$ w.r.t. $n$ )

$$
\exists \chi^{1} \forall x_{1}, x_{2} \leq_{1} \nu^{d}(M, \ldots, M) \forall k^{0}\left(\left\|\tilde{x}_{1}-\mathbb{R}^{d} \tilde{x}_{2}\right\|_{\max } \leq \frac{1}{\chi^{k+1}} \rightarrow\left|\Phi \tilde{x}_{1}-\mathbb{R} \Phi \tilde{x}_{2}\right|_{\mathbb{R}}<\frac{1}{k+1}\right) .
$$

2) Using $\Sigma_{1}^{0}-\mathrm{UB}^{-}$instead of $\Sigma_{1}^{0}$-UB in the proof of 1) one obtains

$$
\left\{\begin{array}{l}
\exists \chi^{1} \forall x_{1}, x_{2} \leq_{1} \nu^{d}(M, \ldots, M) \forall l^{0}, k^{0}\left(\| \widetilde{\widetilde{\left(x_{1}, l\right.}}\right)--\left._{\mathbb{R}^{d}}\left(\widetilde{\overline{x_{2}, l}}\right)\right|_{\max } \leq \frac{1}{\chi^{k+1}} \rightarrow \\
\left.\left|\Phi\left(\overline{\overline{x_{1}, l}}\right)-{ }_{\mathbb{R}} \Phi\left(\widetilde{\widetilde{x_{2}, l}}\right)\right|_{\mathbb{R}}<\frac{1}{k+1}\right) .
\end{array}\right.
$$

\footnotetext{
${ }^{6}$ Here $\tilde{x}$ is a shortage for $\nu^{d}\left(\widetilde{\nu_{1}^{d}\left(x_{1}\right)}, \ldots, \widetilde{\nu_{d}^{d}\left(x_{d}\right)}\right)$. Since real numbers are represented as Cauchy sequences with fixed rate of convergence, $\leq \leq_{\mathbb{R}} \in \Pi_{1}^{0}$ and $<_{\mathbb{R}} \in \Sigma_{1}^{0}$; see [12] for details.

${ }^{7}$ Instead of $\|\cdot\|_{\max }$ we can also use e.g. the Euclidian metric on $\mathbb{R}^{d}$ thereby obtaining a modulus of continuity w.r.t. this metric. However, since both norms on $\mathbb{R}^{d}$ are constructively equivalent, a modulus of uniform continuity w.r.t. one norm can be easily transformed into a modulus for the other norm.
} 
Since $\left\|(\widetilde{\overline{x, l}})-\mathbb{R}^{d} \tilde{x}\right\|_{\max } \leq \frac{2}{k+1}$ for $l>3(k+1)$, this together with the pointwise continuity of $\Phi$ implies the claim.

This result generalizes also to variable rectangles $\left[a_{1}, b_{1}\right] \times \ldots \times\left[a_{d}, b_{d}\right]$ instead of $[0,1]^{d}$ (where $a_{i}<b_{i}$ for $\left.i=1, \ldots, d\right)$.

Corollary $3.2 G_{2} A^{\omega} \oplus \Sigma_{1}^{0}-U B$ (and therefore $G_{2} A^{\omega} \oplus A C^{1,0}-q f \oplus F$ ) proves: Every $\Phi^{1(1)}$ which represents an unrestricted function $\mathbb{R}^{d} \rightarrow \mathbb{R}$ is pointwise continuous on $\mathbb{R}^{d}$ and possesses a modulus of pointwise continuity operation.

Proof: ¿From the proof of 1) above we obtain a function $\chi^{1(0)}$ such that $\chi(m)$ is a modulus of uniform continuity for $\Phi$ on $[-m, m]^{d}$ by applying $\Sigma_{1}^{0}-\mathrm{UB}$ to

$\forall m^{0} \forall x_{1}, x_{2} \leq{ }_{1} \nu^{d}(M(m), \ldots, M(m)) \forall k^{0} \exists n(\underbrace{\left\|x_{1}-\mathbb{R}^{d} x_{2}\right\|_{\max } \leq \frac{1}{n+1} \rightarrow\left|\Phi x_{1}-\mathbb{R} \Phi x_{2}\right|_{\mathbb{R}}<d \frac{1}{k+1}}_{\in \Sigma_{1}^{0}})$,

where $M(m):=\lambda n \cdot j(6 m(n+1), 3(n+1)-1)$ is the boundedness function from our representation of $[-m, m]$.

Now define $\xi^{0(1)} \in \mathrm{G}_{2} \mathrm{R}^{\omega}$ by $\left.\xi\left(x^{1}\right):=\max _{0}\left(\left\lceil\left(\nu_{1}^{\widehat{d}(x)}\right)(1)\right\rceil+2, \ldots,\left\lceil\left(\nu_{d}^{d(x}\right)\right)(1)\right\rceil+2\right)$. The natural number $\xi\left(x^{1}\right)$ is an upper bound for $\left\|x^{1}\right\|_{\max }+1$. Hence $\omega x^{1}:=\lambda k^{0} \cdot \chi(\xi(x), k)$ is a modulus of pointwise continuity in $x$, since $\|x-y\|_{\max } \leq \frac{1}{\omega(x, k)+1}$ implies that $\|x\|_{\max },\|y\|_{\max } \leq \xi(x)$.

Remark 3.3 The modulus of pointwise continuity $\omega\left(x^{1}, k^{0}\right)$ is only an operation and not a function of $x$ as an element of $\mathbb{R}^{d}$ (but a function of $x \in \mathbb{N}^{\mathbb{N}}$ as an representative of such an element) since it is not extensional w.r.t. $=_{\mathbb{R}^{d}}$.

\section{Application 2: Sequential form of the Heine-Borel covering property of $[0,1]^{d}$ and other compact spaces}

Let $B_{\varepsilon}\left(x_{0}\right):=\left\{y \in \mathbb{R}^{d}:\left\|x_{0}-y\right\|_{E}<\varepsilon\right\}$ denote the open ball with centre $x_{0} \in \mathbb{R}^{d}$ and radius $\varepsilon$ ( w.r.t. the Euclidian norm).

Proposition $3.4 G_{2} A_{i}^{\omega} \oplus \Sigma_{1}^{0}-U B^{-}$(and therefore $G_{2} A^{\omega} \oplus A C^{1,0}-q f \oplus F^{-}$) proves that every sequence of open balls which cover $[0,1]^{d}$ contains a finite sub-cover.

Proof: We have to show

$$
\text { (1) }\left\{\begin{aligned}
\forall f: \mathbb{N} \rightarrow \mathbb{R}_{+} \backslash\{0\} \forall g: \mathbb{N} \rightarrow[0,1]^{d}\left(\forall x \in[0,1]^{d} \exists k \in \mathbb{N}\left(x \in B_{f k}(g k)\right)\right. \\
\left.\rightarrow \exists k_{0} \forall x \in[0,1]^{d} \exists k \leq k_{0}\left(x \in B_{f k}(g k)\right)\right) .
\end{aligned}\right.
$$

When formalized in $\mathrm{G}_{2} \mathrm{~A}_{i}^{\omega}(1)$ has the form (compare application 1 above)

$$
\left\{\begin{aligned}
\forall f^{1(0)}, g^{1(0)}\left(\forall l^{0}(f l\right. & \left.>_{\mathbb{R}} 0\right) \wedge \forall x \leq_{1} \nu^{d}(M, \ldots, M) \exists k^{0}\left(\left\|\tilde{x}-\mathbb{R}^{d} g k\right\|_{E}<\mathbb{R} f k\right) \\
\rightarrow & \left.\exists k_{0}^{0} \forall x \leq_{1} \nu^{d}(M, \ldots, M) \exists k \leq_{0} k_{0}\left(\left\|\tilde{x}-{ }_{\mathbb{R}^{d}} g k\right\|_{E}<\mathbb{R} f k\right)\right)
\end{aligned}\right.
$$


Using $\Sigma_{1}^{0}-\mathrm{UB}^{-}$and the fact that $<_{\mathbb{R}} \in \Sigma_{1}^{0}$ we obtain

(3) $\left\{\begin{aligned} \forall f^{1(0)}, g^{1(0)}\left(\forall l^{0}\left(f l>_{\mathbb{R}} 0\right) \wedge \forall x \leq_{1} \nu^{d}(M, \ldots, M) \exists k^{0}\left(\left\|\tilde{x}-\mathbb{R}_{\mathbb{R}^{d}} g k\right\|_{E}<\mathbb{R} f k\right)\right. \\ \left.\rightarrow \exists k_{0}^{0} \forall x \leq_{1} \nu^{d}(M, \ldots, M) \forall n^{0} \exists k, m \leq_{0} k_{0}\left(\left\|(\widetilde{\overline{x, n}})-\mathbb{R}_{\mathbb{R}^{d}} g k\right\|_{E}<\mathbb{R} f k-\frac{1}{m+1}\right)\right) .\end{aligned}\right.$

Since $\left\|(\widetilde{\overline{x, n}})-{ }_{\mathbb{R}^{d}} \tilde{x}\right\|_{\max } \leq \frac{2}{k+1}$ for $n>3(k+1)$, (3) implies $(2)$ which concludes the proof.

Similarly one shows this result for $\left[a_{1}, b_{1}\right] \times \ldots \times\left[a_{d}, b_{d}\right]$ and also for other compact spaces as e.g. $K_{c, \lambda}:=\left\{f \in C[0,1]:\|f\|_{\infty} \leq c \wedge f\right.$ has Lipschitz constant $\left.\lambda\right\}$.

Application 3: Attainment of the maximum value for $f \in C\left([0,1]^{d}, \mathbb{R}\right)$

Proposition 3.5 1) $G_{2} A^{\omega} \oplus \Sigma_{1}^{0}-U B$ (and therefore $G_{2} A^{\omega} \oplus A C^{1,0}-q f \oplus F$ ) proves:

Every function $F:[0,1]^{d} \rightarrow \mathbb{R}$ attains it maximum value on $[0,1]^{d}$.

2) $G_{2} A^{\omega} \oplus \Sigma_{1}^{0}-U B^{-}$(and therefore $G_{2} A^{\omega} \oplus A C^{1,0}-q f \oplus F^{-}$) proves:

Every pointwise continuous function $F:[0,1]^{d} \rightarrow \mathbb{R}$ attains it maximum value on $[0,1]^{d}$.

Proof: In view of prop.3.1 and the remark to its proof we only have to show 2). Assume

(1) $\exists \Phi:[0,1]^{d} \rightarrow \mathbb{R}\left(\Phi\right.$ pointwise cont. $\left.\wedge \forall x \in[0,1]^{d} \exists r \in[0,1]^{d} \cap \mathbb{Q}^{d}(\Phi x<\Phi r)\right)$.

The proposition $\forall x \in[0,1]^{d} \exists r \in[0,1]^{d} \cap \mathbb{Q}^{d}(\Phi x<\Phi r)$ has the following logical form

$$
\text { (2) } \forall x \leq_{1} \nu^{d}(M, \ldots, M) \underbrace{\exists n^{0}\left(\Phi \tilde{x}<_{\mathbb{R}} \Phi\left(\lambda k^{0} . q(n)\right)\right.}_{\in \Sigma_{1}^{0}}),
$$

where $q \in \mathrm{G}_{2} \mathrm{R}^{\omega}$ is an enumeration of $[0,1]^{d} \cap \mathbb{Q}^{d}$. (2) implies

$$
\text { (3) } \forall x \leq_{1} \nu^{d}(M, \ldots, M) \exists n^{0}, l^{0}\left(\Phi \tilde{x}<\mathbb{R} \Phi\left(\lambda k^{0} \cdot q(n)\right)-\frac{1}{l+1}\right) .
$$

$\Sigma_{1}^{0}-\mathrm{UB}^{-}$applied to (3) yields

$$
\exists n_{0} \forall x \leq_{1} \nu^{d}(M, \ldots, M) \forall l^{0} \exists n \leq_{0} n_{0}\left(\Phi(\widetilde{\overline{x, l}})<_{\mathbb{R}} \Phi\left(\lambda k^{0} \cdot q(n)\right)-\frac{1}{n_{0}+1}\right) .
$$

In $\mathrm{G}_{2} \mathrm{~A}^{\omega}$ one can show that there exists an $n_{1} \leq n_{0}$ be such that

$$
\text { (4) }\left|\Phi\left(\lambda k^{0} \cdot q\left(n_{1}\right)\right)-\mathbb{R}_{\mathbb{R}} \max _{\mathbb{R}}\left(\Phi\left(\lambda k^{0} \cdot q(0)\right), \ldots, \Phi\left(\lambda k^{0} \cdot q\left(n_{0}\right)\right)\right)\right|<_{\mathbb{R}} \frac{1}{2\left(n_{0}+1\right)} .
$$

Since for all $m^{0}$ there exist $x^{1}, l^{0}$ such that $x \leq_{1} \nu^{d}(M, \ldots, M)$ and $\left.\mid \widetilde{\overline{x, l}}\right)-\mathbb{R}_{\mathbb{R}} \lambda k^{0} \cdot q\left(n_{1}\right) \mid<\mathbb{R} \frac{1}{m+1}$ we obtain (using the pointwise continuity of $\Phi$ ) a contradiction to (1). Hence

(5) $\forall \Phi:[0,1]^{d} \rightarrow \mathbb{R}\left(\Phi\right.$ pointwise cont. $\left.\rightarrow \exists x \in[0,1]^{d} \forall r \in[0,1]^{d} \cap \mathbb{Q}^{d}(\Phi x \geq \Phi r)\right)$,

which implies

(6) $\forall \Phi:[0,1]^{d} \rightarrow \mathbb{R}\left(\Phi\right.$ pointwise continuous $\left.\rightarrow \exists x \in[0,1]^{d} \forall y \in[0,1]^{d}(\Phi x \geq \Phi y)\right)$. 
Remark 3.6 In [8] we have shown that the statement expressing the attainment of the maximum of uniformly continuous functions on $[0,1]^{d}$ can be expressed as an axiom $\Delta$ and therefore it is not necessary to prove it using $\Sigma_{1}^{0}-U B^{-}$. However expressing the statement in this form requires a very complicated representation of the elements of the space $C\left([0,1]^{d}, \mathbb{R}\right)$ which is avoided altogether in our proof above.

\section{Application 4: Dini's theorem}

Proposition 3.7 1) $G_{2} A_{i}^{\omega} \oplus \Sigma_{1}^{0}-U B$ (and therefore $G_{2} A^{\omega} \oplus A C^{1,0}-q f \oplus F$ ) proves: Every sequence $\Phi_{n}$ of functions $:[0,1]^{d} \rightarrow \mathbb{R}$ which increases pointwise to a function $\Phi:[0,1]^{d} \rightarrow \mathbb{R}$ converges uniformly on $[0,1]^{d}$ to $\Phi$, and there exists a modulus of uniform convergence.

2) $G_{2} A_{i}^{\omega} \oplus \Sigma_{1}^{0}-U B^{-}$(and therefore $G_{2} A^{\omega} \oplus A C^{1,0}-q f \oplus F^{-}$) proves: Every sequence $\Phi_{n}$ of pointwise continuous functions : $[0,1]^{d} \rightarrow \mathbb{R}$ which increases pointwise to a pointwise continuous function $\Phi:[0,1]^{d} \rightarrow \mathbb{R}$ converges uniformly on $[0,1]^{d}$ to $\Phi$, and there exists a modulus of uniform convergence.

Proof: By the assumption we have

$$
\forall k^{0} \forall x \in[0,1]^{d} \exists n^{0}\left(\Phi x-\Phi_{n} x<\mathbb{R} \frac{1}{k+1}\right) .
$$

Similarly to the proof of prop.3.5 one obtains using $\Sigma_{1}^{0}-\mathrm{UB}$

$$
\exists \chi^{1} \forall k^{0} \forall x \in[0,1]^{d} \exists n \leq_{0} \chi(k)\left(\Phi x-\Phi_{n} x<\mathbb{R} \frac{1}{k+1}\right) .
$$

Since $\left(\Phi_{n}\right)_{n \in \mathbb{N}}$ is increasing this implies

$$
(*) \exists \chi^{1} \forall k^{0} \forall x \in[0,1]^{d} \forall n \geq_{0} \chi(k)\left(\Phi x-\Phi_{n} x<\mathbb{R} \frac{1}{k+1}\right),
$$

which concludes the proof of 1$)$.

By $\Sigma_{1}^{0}-\mathrm{UB}^{-}$we obtain $(*)$ only for a dense subset of $[0,1]^{d}$. However this implies $(*)$ if $\Phi_{n}, \Phi$ are assumed to be pointwise continuous on $[0,1]^{d}$.

\section{Application 5: Existence of the inverse function of a strictly monotone function}

Proposition $3.8 \quad 1$ ) $G_{2} A^{\omega} \oplus \Sigma_{1}^{0}-U B$ (and therefore $G_{2} A^{\omega} \oplus A C^{1,0}-q f \oplus F$ ) proves:

Every strictly increasing function $\Phi:[0,1] \rightarrow \mathbb{R}$ possesses a strictly increasing inverse function $\Phi^{-1}:[\Phi 0, \Phi 1] \rightarrow[0,1]$ which is uniformly continuous on $[\Phi 0, \Phi 1]$ and has a modulus of uniform continuity.

2) $G_{2} A^{\omega} \oplus \Sigma_{1}^{0}-U B^{-}$(and therefore $G_{2} A^{\omega} \oplus A C^{1,0}-q f \oplus F^{-}$) proves:

Every strictly increasing pointwise continuous function $\Phi:[0,1] \rightarrow \mathbb{R}$ possesses a strictly increasing inverse function $\Phi^{-1}:[\Phi 0, \Phi 1] \rightarrow[0,1]$ which is uniformly continuous on $[\Phi 0, \Phi 1]$ and has a modulus of uniform continuity. 
Proof: The strict monotonicity of $\Phi$ implies

$$
\text { (1) } \forall x, y \in[0,1] \forall k^{0} \exists n^{0}\left(x \geq y+\frac{1}{k+1} \rightarrow \Phi x>\Phi y+\frac{1}{n+1}\right) .
$$

Modulo our representation of $[0,1], \Phi$ and $\geq_{\mathbb{R}},>_{\mathbb{R}}$ (1) has the logical form

$$
\forall x, y \leq_{1} M \forall k^{0} \exists n^{0}(\underbrace{\tilde{x} \geq_{\mathbb{R}} \tilde{y}+\mathbb{R} \frac{1}{k+1} \rightarrow \Phi \tilde{x}>_{\mathbb{R}} \Phi \tilde{y}+\frac{1}{n+1}}_{\equiv: A \in \Sigma_{1}^{0}}) .
$$

By $\Sigma_{1}^{0}$-UB we obtain (using the monotonicity of $A$ w.r.t. $n$ ) a modulus of uniform strict monotonicity, i.e.

$$
\text { (2) } \exists \chi^{1} \forall x, y \leq_{1} M \forall k^{0}\left(\tilde{x} \geq_{\mathbb{R}} \tilde{y}+\mathbb{R} \frac{1}{k+1} \rightarrow \Phi \tilde{x}>_{\mathbb{R}} \Phi \tilde{y}+\frac{1}{\chi k+1}\right)
$$

(If we use $\Sigma_{1}^{0}-\mathrm{UB}^{-}$only instead of $\Sigma_{1}^{0}-\mathrm{UB}$ we obtain the restriction of (2) to a dense subset of $[0,1]$ which implies (2) if $\Phi$ is assumed to be pointwise continuous).

The rest of the proof is now straightforward and left to the reader.

The applications $1-5$ show that $\Sigma_{1}^{0}-\mathrm{UB}^{-}$and hence $F^{-}$combined with $\mathrm{AC}^{1,0}-\mathrm{qf}$ allows to give very short proofs for important theorems in analysis. In these proofs one can treat continuous functions

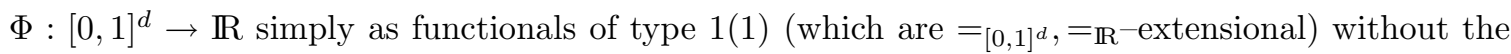
need of the quite complicated representation of $C\left([0,1]^{d}, \mathbb{R}\right)$ from chapter 3 of [8]. Moreover the applications 1-4 generalize to other compact spaces $K$ instead of $[0,1]^{d}$ as long as the elements of $K$ can be represented by $\left\{f^{1}: f \leq_{1} t\right\}$ for a suitable term $t$.

\section{References}

[1] Bezem, M.A., Strongly majorizable functional of finite type: a model for bar recursion containing discontinuous functionals. J. Symb. Logic 50, pp. 652-660 (1985).

[2] Feferman, S., Theories of finite type related to mathematical practice. In: Barwise, J. (ed.), Handbook of Mathematical Logic, North-Holland, Amsterdam, pp. 913-972 (1977).

[3] Friedman, H., Systems of second order arithmetic with restricted induction (abstract), J. Symbolic Logic 41, pp. 558-559 (1976).

[4] Kohlenbach, U., Effective bounds from ineffective proofs in analysis: an application of functional interpretation and majorization. J. Symbolic Logic 57, pp. 1239-1273 (1992).

[5] Kohlenbach, U., Effective moduli from ineffective uniqueness proofs. An unwinding of de La Vallée Poussin's proof for Chebycheff approximation. Ann. Pure Appl. Logic 64, pp. 27-94 (1993).

[6] Kohlenbach, U., New effective moduli of uniqueness and uniform a-priori estimates for constants of strong unicity by logical analysis of known proofs in best approximation theory. Numer. Funct. Anal. and Optimiz. 14, pp. 581-606 (1993). 
[7] Kohlenbach, U., Analysing proofs in analysis. In: W. Hodges, M. Hyland, C. Steinhorn, J. Truss, editors, Logic: from Foundations to Applications. European Logic Colloquium (Keele, 1993), pp. 225-260, Oxford University Press (1996).

[8] Kohlenbach, U., Real growth in standard parts of analysis. Habilitationsschrift, xv+166 p., Frankfurt (1995).

[9] Kohlenbach, U., Mathematically strong subsystems of analysis with low rate of provably recursive functionals. Arch. Math. Logic 36, pp. 31-71 (1996).

[10] Kohlenbach, U., Relative constructivity. To appear in: J. Symbolic Logic.

[11] Kohlenbach, U., Elimination of Skolem functions for monotone formulas. To appear in: Arch. Math. Logic.

[12] Kohlenbach, U., Arithmetizing proofs in analysis. To appear in: Proc. Logic Colloquium 96 (San Sebastian).

[13] Luckhardt, H., Extensional Gödel functional interpretation. A consistency proof of classical analysis. Springer Lecture Notes in Mathematics 306 (1973).

[14] Sieg, W., Fragments of arithmetic. Ann. Pure Appl. Logic 28, pp. 33-71 (1985).

[15] Simpson, S.G., Which set existence axioms are needed to prove the Cauchy/Peano theorem for ordinary differential equations. J. Symbolic Logic 49,pp. 783-801 (1984).

[16] Simpson, S.G., Reverse Mathematics. Proc. Symposia Pure Math. 42, pp. 461-471, AMS, Providence (1985).

[17] Troelstra, A.S., Note on the fan theorem. J. Symbolic Logic 39, pp. 584-596 (1974). 